\title{
HARMONIC UNIVALENT CONVEX FUNCTIONS USING A QUANTUM CALCULUS APPROACH
}

\author{
O. P. Ahuja, A. Çetinkaya, Y. PolatoĞLu
}

ABstract. In this paper, we make an attempt to use quantum calculus and harmonic univalent convex functions. In particular, we introduce and investigate the properties of $q$-harmonic functions and $q$-harmonic convex functions of order $\alpha$.

2010 Mathematics Subject Classification: 30C45, 30C50, 30C99, 81Q99.

Keywords: Quantum calculus, $q$-difference operator, $q$-derivative operator, $q$-calculus, $q$-harmonic function, $q$-harmonic convex function of order $\alpha$, convolution.

\section{INTRODUCTION}

After the development of quantum calculus, popularly known as $q$-calculus, by Jackson (see [14, 15, 16]) in 1909-1910, quantum calculus has been applied in physics, quantum mechanics, analytic number theory, sobolev spaces, group representation theory, theta functions, gamma functions, operator theory and several other areas. For the definitions and properties of $q$-calculus, one may refer to $[5,6,9,10,11,18]$.

In 1990, Ismail, Merkes and Styer [13] used $q$-calculus in the theory of analytic univalent functions by defining a class of complex-valued functions that are analytic on the open unit disc $\mathbb{D}:=\{z:|z|<1\}$ with the normalizations $f(0)=0, f^{\prime}(0)=1$, and $|f(q z)| \leq|f(z)|$ on $\mathbb{D}$ for every $q, q \in(0,1)$. Motivated by these authors, several researchers used the theory of analytic univalent functions and $q$-calculus; for example see [4, 20, 23].

Though the theory of harmonic univalent functions was discovered by Clunie and Sheil-Small in 1984 [7], but the role of $q$-calculus in this specialized area of research is quite new. Motivated by a method for $q$-calculus, outlined by Ernst [9] in 2003, we in this paper make an attempt to apply $q$-calculus and harmonic convex functions. In particular, we introduce and study a class of generalized harmonic univalent convex functions by using $q$-calculus approach. 


\section{Preliminaries}

Throughout this paper, we shall assume that $q$-satisfies the condition $q \in(0,1)$.

Definition 1. Let $q \in(0,1)$ and $\lambda \in \mathbb{C}$. The $q$-number, denoted by $[\lambda]_{q}$, is defined by

$$
[\lambda]_{q}=\frac{1-q^{\lambda}}{1-q} .
$$

When $\lambda=n \in \mathbb{N}$, we obtain $[n]_{q}=1+q+q^{2}+\ldots+q^{n-1}$, and when $q \rightarrow 1^{-}$, then $[n]_{q}=n$.

Applying the above $q$-number and motivated by Jackson [14], $q$-derivative is defined below.

Definition 2. The $q$-derivative (or $q$-difference operator) of a function $f$, defined on a subset of $\mathbb{C}$, is given by

$$
\left(D_{q} f\right)(z)=\left\{\begin{array}{cc}
\frac{f(z)-f(q z)}{(1-q) z}, & z \neq 0 \\
f^{\prime}(0), & z=0
\end{array}\right.
$$

We note that $\lim _{q \rightarrow 1^{-}}\left(D_{q} f\right)(z)=f^{\prime}(z)$ if $f$ is differentiable at $z$.

For definitions and properties of $q$-derivative and $q$-calculus, one may refer to $[5,10,11,18]$.

For a function $f(z)=z^{n}$, we observe that

$$
D_{q} z^{n}=\frac{1-q^{n}}{1-q} z^{n-1}=[n]_{q} z^{n-1} .
$$

Let $\mathcal{A}$ denote the class of normalized functions of the form

$$
f(z)=\sum_{n=1}^{\infty} a_{n} z^{n}, a_{1}=1
$$

which are analytic in the open unit disk $\mathbb{D}$. In view of $(1)$ and $(2)$, it follows that for any $f \in \mathcal{A}$, we have

$$
D_{q} f(z)=\sum_{n=1}^{\infty}[n]_{q} a_{n} z^{n-1} \quad \text { and } \quad D_{q}\left(z D_{q} f(z)\right)=\sum_{n=1}^{\infty}[n]_{q}^{2} a_{n} z^{n-1},
$$

where $q \in(0,1)$. 
Jackson [15] introduced the $q$-integral of a function $f$ as

$$
\int_{0}^{z} f(t) d_{q} t=z(1-q) \sum_{n=0}^{\infty} q^{n} f\left(z q^{n}\right)
$$

provided the series converges.

In order to use $q$-calculus and harmonic univalent functions, we first need some notations and terminology of harmonic univalent functions.

Let $\mathcal{H}$ denote the family of continuous complex-valued sense-preserving functions $f=h+\bar{g}$ in the unit disc $\mathbb{D}$ that are harmonic and where

$$
h(z)=z+\sum_{n=2}^{\infty} a_{n} z^{n}, \quad g(z)=\sum_{n=1}^{\infty} b_{n} z^{n} \quad\left|b_{1}\right|<1 .
$$

Note that $f=h+\bar{g}$ is locally univalent and sense-preserving in $\mathbb{D}$ if and only if $\left|g^{\prime}(z) / h^{\prime}(z)\right|<1$ in $\mathbb{D}$ (see [7]). Also, let $\mathcal{S}_{\mathcal{H}}$ be a subclass of functions $f$ in $\mathcal{H}$ that are univalent in $\mathbb{D}$. We observe that for $g(z) \equiv 0$ in $\mathbb{D}$, the class $\mathcal{S}_{\mathcal{H}}$ reduces to the class $\mathcal{S}$ of normalized analytic univalent functions in $\mathbb{D}$.

We also recall that convolution of two complex-valued harmonic functions

$$
f_{1}(z)=z+\sum_{n=2}^{\infty} a_{1 n} z^{n}+\sum_{n=1}^{\infty} \overline{b_{1 n} z^{n}} \quad \text { and } \quad f_{2}(z)=z+\sum_{n=2}^{\infty} a_{2 n} z^{n}+\sum_{n=1}^{\infty} \overline{b_{2 n} z^{n}}
$$

is defined by

$$
f_{1}(z) * f_{2}(z)=\left(f_{1} * f_{2}\right)(z)=z+\sum_{n=2}^{\infty} a_{1 n} a_{2 n} z^{n}+\sum_{n=1}^{\infty} \overline{b_{1 n} b_{2 n} z^{n}}, \quad(z \in \mathbb{D}) .
$$

A comprehensive study for the theory of harmonic univalent functions may be found in Duren [8]. One may also refer to the survey articles by the first author [1] and $[2]$.

We now define the class $\mathcal{H}_{q}$ consisting of $q$-harmonic functions in $\mathbb{D}$.

Definition 3. A harmonic function $f=h+\bar{g}$ defined by (3) is said to be $q$-harmonic, locally univalent and sense-preserving in $\mathbb{D}$ denoted by $\mathcal{H}_{q}$, if and only if the second dilatation $w_{q}$ satisfies the condition

$$
\left|w_{q}(z)\right|=\left|\frac{D_{q} g(z)}{D_{q} h(z)}\right|<1
$$

where $q \in(0,1)$ and $z \in \mathbb{D}$. Note that as $q \rightarrow 1^{-}, \mathcal{H}_{q}$ reduces to the family $\mathcal{H}$. 
O. P. Ahuja, A. Çetinkaya, Y. Polatoğlu - Harmonic Univalent Convex ...

We also define a new operator and we call it as $q$-Bernardi harmonic integral operator.

Definition 4. Let $f=h+\bar{g} \in \mathcal{H}_{q}$ be defined by (3). Then the operator $L: \mathcal{H} \rightarrow \mathcal{H}$ is said to be $q-$ Bernardi harmonic integral operator denoted by $L(f)=F$ defined as

$$
F(z)=\frac{[c+1]_{q}}{z^{c}} \int_{0}^{z} t^{c-1} h(t) d_{q} t+\overline{\frac{[c+1]_{q}}{z^{c}} \int_{0}^{z} t^{c-1} g(t) d_{q} t}, \quad c=1,2, \ldots .
$$

Note that for $q \rightarrow 1^{-}$, this operator reduces to the operator studied for a class defined by fractional operator in [21]. Letting $g(z) \equiv 0$ for all $z \in \mathbb{D}$, the operator (5) gives the corresponding operator introduced in [20]. The operator defined by (5) is named after Bernardi because by letting $q \rightarrow 1^{-}$and $g(z) \equiv 0$, we obtain the operator $I: \mathcal{A} \rightarrow \mathcal{A}$ defined by

$$
I(z)=\frac{c+1}{z^{c}} \int_{0}^{z} t^{c-1} f(t) d t .
$$

In fact, for $q \rightarrow 1^{-}, g(z) \equiv 0$ and $c=1$, our operator (5) reduces to the Libera integral operator defined and studied by Libera [19].

In this paper, we introduce and study the class $\mathcal{S}_{\mathcal{H}_{q}}^{\mathcal{C}}(\alpha)$ defined as below.

Definition 5. A function $f=h+\bar{g}$ in $\mathcal{H}_{q}$ is said to be in $\mathcal{S}_{\mathcal{H}_{q}}^{\mathcal{C}}(\alpha)$ if

$$
\operatorname{Re}\left(\frac{z D_{q}\left(z D_{q} h(z)\right)+\overline{z D_{q}\left(z D_{q} g(z)\right)}}{z D_{q} h(z)-\overline{z D_{q} g(z)}}\right)>\alpha, \quad|z|=r<1,
$$

where $q \in(0,1)$ and $\alpha \in[0,1)$. A function $f$ in $\mathcal{S}_{\mathcal{H}_{q}}^{\mathcal{C}}(\alpha)$ is called $q$-harmonic convex function of order $\alpha$.

Remark 1. When $q \rightarrow 1^{-}$, then the class $\mathcal{S}_{\mathcal{H}_{q}}^{\mathcal{C}}(\alpha)$ reduces to the traditional class $\mathcal{S}_{\mathcal{H}}^{\mathcal{C}}(\alpha)$, known as harmonic convex functions of order $\alpha$ (see for example [1, 2, 8]). Also, note that when $q \rightarrow 1^{-}$and $g(z) \equiv 0$ in $\mathbb{D}$, then the class $\mathcal{S}_{\mathcal{H}_{q}}^{\mathcal{C}}(\alpha)$ reduces to the traditional class $\mathcal{S}^{\mathcal{C}}(\alpha)$, known as convex functions of order $\alpha$, was studied by Robertson in 1936 [22]. Moreover, if $g(z) \equiv 0$ in $\mathbb{D}$, then $\mathcal{S}_{\mathcal{H}_{q}}^{\mathcal{C}}(\alpha) \equiv \mathcal{S}_{q}^{\mathcal{C}}(\alpha)$, [23].

\section{Coefficient Bounds and Univalence Criteria}

We begin with a theorem that relates the modulus of the coefficients to the $q$-harmonic class $\mathcal{H}_{q}$. 
Theorem 1. Let $f=h+\bar{g}$ be given by (3). Also, suppose

$$
\sum_{n=1}^{\infty}\left([n]_{q} \frac{[n]_{q}-\alpha}{1-\alpha}\left|a_{n}\right|+[n]_{q} \frac{[n]_{q}+\alpha}{1-\alpha}\left|b_{n}\right|\right) \leq 2,
$$

where $a_{1}=1, \alpha \in[0,1)$ and $q \in(0,1)$. Then $f$ is locally univalent and sensepreserving in $\mathbb{D}$; that is $f \in \mathcal{H}_{q}$.

Proof. Since

$$
\begin{aligned}
\left|D_{q} h(z)\right| & =\left|1-\sum_{n=1}^{\infty}[n]_{q} a_{n} z^{n-1}\right| \\
& >1-\sum_{n=1}^{\infty}[n]_{q}\left|a_{n}\right| \\
& \geq 1-\sum_{n=1}^{\infty}[n]_{q} \frac{[n]_{q}-\alpha}{1-\alpha}\left|a_{n}\right| \\
& \geq \sum_{n=1}^{\infty}[n]_{q} \frac{[n]_{q}+\alpha}{1-\alpha}\left|b_{n}\right| \\
& \geq \sum_{n=1}^{\infty}[n]_{q}\left|b_{n}\right| \\
& >\sum_{n=1}^{\infty}[n]_{q}\left|b_{n}\right||z|^{n-1} \geq\left|D_{q} g(z)\right|,
\end{aligned}
$$

it follows that $f \in \mathcal{H}_{q}$, by Definition 3 .

The following theorem gives necessary and sufficient convolution condition for $q$-harmonic convex functions of order $\alpha$.

Theorem 2. Let $f=h+\bar{g}$ with $h$ and $g$ of the form (3), where $f$ belongs to $\mathcal{H}_{q}$. Then $f \in \mathcal{S}_{\mathcal{H}_{q}}^{\mathcal{C}}(\alpha)$ if and only if

$$
H_{1}(z)+\overline{G_{1}}(z) \neq 0,
$$

where

$$
\begin{gathered}
H_{1}(z)=h(z) * \frac{(2-2 \alpha) z+[2-2 \alpha+(2 \alpha+\zeta-1)(1+q)] q z^{2}}{(1-z)(1-q z)\left(1-q^{2} z\right)} \\
\overline{G_{1}}(z)=\overline{g(z)} * \frac{(2 \zeta+2 \alpha) \bar{z}+[2 \zeta+2 \alpha-(2 \alpha+\zeta-1)(1+q)] q \bar{z}^{2}}{(1-\bar{z})(1-q \bar{z})\left(1-q^{2} \bar{z}\right)}
\end{gathered}
$$

$|\zeta|=1, \zeta \neq-1, \alpha \in[0,1), q \in(0,1)$ and $0<|z|<1$. 
Proof. Since at $z=0$

$$
\frac{z D_{q}\left(z D_{q} h(z)\right)+\overline{z D_{q}\left(z D_{q} g(z)\right)}}{z D_{q} h(z)-\overline{z D_{q} g(z)}}=1,
$$

the required condition (6) is equivalent to

$$
\frac{\frac{z D_{q}\left(z D_{q} h(z)\right)+\overline{z D_{q}\left(z D_{q} g(z)\right)}}{z D_{q} h(z)-z D_{q} g(z)}-\alpha}{1-\alpha} \neq \frac{\zeta-1}{\zeta+1} .
$$

It is straight forward to verify that

$$
f(z) * \frac{z}{1-z}=f(z) \text { and } f(z) * \frac{z}{(1-z)(1-q z)}=z D_{q} f(z) .
$$

Using (9), (10) and algebraic manipulations, we get

$$
\begin{aligned}
& 0 \neq(\zeta+1)\left[z D_{q}\left(z D_{q} h(z)\right)+\overline{z D_{q}\left(z D_{q} g(z)\right)}-\alpha z D_{q} h(z)+\alpha \overline{z D_{q} g(z)}\right] \\
& \quad-(\zeta-1)\left[z D_{q} h(z)-\overline{z D_{q} g(z)}-\alpha z D_{q} h(z)+\alpha \overline{z D_{q} g(z)}\right]:=H_{2}(z)+\overline{G_{2}}(z),
\end{aligned}
$$

where

$$
\begin{aligned}
H_{2}(z) & =z D_{q} h(z) *\left(\frac{(\zeta+1) z}{(1-z)(1-q z)}-\frac{\alpha(\zeta+1) z}{1-z}-\frac{(\zeta-1) z}{1-z}+\frac{\alpha(\zeta-1) z}{1-z}\right) \\
& =z D_{q} h(z) *\left(\frac{(2-2 \alpha) z+(2 \alpha+\zeta-1) q z^{2}}{(1-z)(1-q z)}\right) \\
& =h(z) * z D_{q}\left(\frac{(2-2 \alpha) z+(2 \alpha+\zeta-1) q z^{2}}{(1-z)(1-q z)}\right) \\
& =h(z) * \frac{(2-2 \alpha) z+[2-2 \alpha+(2 \alpha+\zeta-1)(1+q)] q z^{2}}{(1-z)(1-q z)\left(1-q^{2} z\right)}=H_{1}(z)
\end{aligned}
$$

and

$$
\begin{aligned}
\overline{G_{2}}(z) & =\overline{z D_{q} g(z)} * \overline{\left(\frac{(\bar{\zeta}+1) z}{(1-z)(1-q z)}+\frac{\alpha(\bar{\zeta}+1) z}{1-z}+\frac{(\bar{\zeta}-1) z}{1-z}-\frac{\alpha(\bar{\zeta}-1) z}{1-z}\right)} \\
& =\overline{z D_{q} g(z)} * \overline{\left(\frac{(2 \bar{\zeta}+2 \alpha) z-(2 \alpha+\bar{\zeta}-1) q z^{2}}{(1-z)(1-q z)}\right)} \\
& =\overline{g(z)} * z D_{q} \frac{\left(\frac{(2 \bar{\zeta}+2 \alpha) z-(2 \alpha+\bar{\zeta}-1) q z^{2}}{(1-z)(1-q z)}\right)}{(2 \bar{\zeta}+2 \alpha) z+[2 \bar{\zeta}+2 \alpha-(2 \alpha+\bar{\zeta}-1)(1+q)] q z^{2}} \\
& =\overline{g(z)} * \frac{G_{1}(z) .}{(1-z)(1-q z)\left(1-q^{2} z\right)}
\end{aligned}
$$

This completes the proof. 
Remark 2. When $q \rightarrow 1^{-}$and $\alpha=0$, Theorem 2 reduces to the corresponding convolution condition obtained in [3].

Theorem 3. Let $f=h+\bar{g}$ with $h$ and $g$ of the form (3). If

$$
\sum_{n=1}^{\infty}\left([n]_{q} \frac{[n]_{q}-\alpha}{1-\alpha}\left|a_{n}\right|+[n]_{q} \frac{[n]_{q}+\alpha}{1-\alpha}\left|b_{n}\right|\right) \leq 2,
$$

where $a_{1}=1, q \in(0,1)$ and $\alpha \in[0,1)$, then $f$ is univalent in $\mathbb{D}$ and $f \in \mathcal{S}_{\mathcal{H}_{q}}^{\mathcal{C}}(\alpha)$.

Proof. In order to prove that $f$ is univalent, we use a technique that was first used by Jahangiri [17] for the univalence of harmonic functions. To this end, we need to show that $f\left(z_{1}\right) \neq f\left(z_{2}\right)$ when $z_{1} \neq z_{2}$. Suppose $z_{1}, z_{2} \in \mathbb{D}$ so that $z_{1} \neq z_{2}$. Since $\mathbb{D}$ is simply connected and convex, we have $z(t)=(1-t) z_{1}+t z_{2} \in \mathbb{D}$, where $0 \leq t \leq 1$. Then we can write

$$
f\left(z_{2}\right)-f\left(z_{1}\right)=\int_{0}^{1}\left[\left(z_{2}-z_{1}\right) D_{q} h(z(t))+\overline{\left(z_{2}-z_{1}\right) D_{q} g(z(t))}\right] d t .
$$

Dividing the above equation by $z_{2}-z_{1} \neq 0$ and taking the real parts, we obtain

$$
\begin{aligned}
\operatorname{Re} \frac{f\left(z_{2}\right)-f\left(z_{1}\right)}{z_{2}-z_{1}} & =\int_{0}^{1} \operatorname{Re}\left[D_{q} h(z(t))+\frac{\overline{\left(z_{2}-z_{1}\right)}}{z_{2}-z_{1}} \overline{D_{q} g(z(t))}\right] d t \\
& >\int_{0}^{1}\left[\operatorname{Re} D_{q} h(z(t))-\left|D_{q} g(z(t))\right|\right] d t \\
& \geq \int_{0}^{1}\left[\operatorname{Re} D_{q} h(z(t))-\sum_{n=1}^{\infty}[n]_{q}\left|b_{n}\right|\right] d t \\
& \geq \int_{0}^{1}\left[1-\sum_{n=2}^{\infty}[n]_{q}\left|a_{n}\right|-\sum_{n=1}^{\infty}[n]_{q}\left|b_{n}\right|\right] d t \\
& \geq \int_{0}^{1}\left[1-\sum_{n=2}^{\infty}[n]_{q} \frac{[n]_{q}-\alpha}{1-\alpha}\left|a_{n}\right|-\sum_{n=1}^{\infty}[n]_{q} \frac{[n]_{q}+\alpha}{1-\alpha}\left|b_{n}\right|\right] d t
\end{aligned}
$$

The last inequality is non-negative by the condition (11). This proves that $f\left(z_{1}\right) \neq$ $f\left(z_{2}\right)$. This leads to the univalence of $f$.

In order to prove that $f \in \mathcal{S}_{\mathcal{H}_{q}}^{\mathcal{C}}(\alpha)$, from (8) we notice

$$
\begin{aligned}
\mid h(z) & *\left(\frac{(2-2 \alpha) z+[2-2 \alpha+(2 \alpha+\zeta-1)(1+q)] q z^{2}}{(1-z)(1-q z)\left(1-q^{2} z\right)}\right) \\
& +\overline{g(z)} * \overline{\left(\frac{(2 \bar{\zeta}+2 \alpha) z+[2 \bar{\zeta}+2 \alpha-(2 \alpha+\bar{\zeta}-1)(1+q)] q z^{2}}{(1-z)(1-q z)\left(1-q^{2} z\right)}\right)} \mid
\end{aligned}
$$




$$
\begin{aligned}
&= \mid(2-2 \alpha)\left[q z^{2} D_{q}\left(D_{q} h(z)\right)+z D_{q} h(z)\right]+(2 \alpha+\zeta-1)\left(q z^{2} D_{q}\left(D_{q} h(z)\right)\right) \\
&+\overline{(2 \bar{\zeta}+2 \alpha)\left[q z^{2} D_{q}\left(D_{q} g(z)\right)+z D_{q} g(z)\right]-(2 \alpha+\bar{\zeta}-1)\left(q z^{2} D_{q}\left(D_{q} g(z)\right)\right)} \mid \\
&= \mid(2-2 \alpha) z+\sum_{n=2}^{\infty}[n]_{q}\left((\zeta+1)[n]_{q}-2 \alpha-(\zeta-1)\right) a_{n} z^{n} \\
&+\sum_{n=1}^{\infty}[n]_{q}\left((\bar{\zeta}+1)[n]_{q}+2 \alpha+(\bar{\zeta}-1)\right) b_{n} z^{n} \mid \\
& \geq(2-2 \alpha)|z|\left[1-\sum_{n=2}^{\infty}[n]_{q}\left|\frac{(\zeta+1)[n]_{q}-2 \alpha-(\zeta-1)}{2-2 \alpha}\right|\left|a_{n}\right||z|^{n-1}\right. \\
&\left.-\sum_{n=1}^{\infty}[n]_{q}\left|\frac{(\bar{\zeta}+1)[n]_{q}+2 \alpha+(\bar{\zeta}-1)}{2-2 \alpha}\right|\left|b_{n}\right||z|^{n-1}\right] \\
&>(2-2 \alpha)|z|\left[1-\sum_{n=2}^{\infty}[n]_{q}\left|\frac{(\zeta+1)[n]_{q}-2 \alpha-(\zeta-1)}{2-2 \alpha}\right|\left|a_{n}\right|\right. \\
&\left.-\sum_{n=1}^{\infty}[n]_{q}\left|\frac{(\bar{\zeta}+1)[n]_{q}+2 \alpha+(\bar{\zeta}-1)}{2-2 \alpha}\right|\left|b_{n}\right|\right], \quad \text { for }|\zeta|=1 \\
&=2(1-\alpha)|z|\left[1-\sum_{n=2}^{\infty}[n]_{q} \frac{[n]_{q}-\alpha}{1-\alpha}\left|a_{n}\right|-\sum_{n=1}^{\infty}[n]_{q} \frac{[n]_{q}+\alpha}{1-\alpha}\left|b_{n}\right|\right] .
\end{aligned}
$$

This last expression is non-negative because of (11). In view of Theorem 2, it follows that $f \in \mathcal{S}_{\mathcal{H}_{q}}^{\mathcal{C}}(\alpha)$.

The $q$ - harmonic convex mappings

$$
f(z)=z+\sum_{n=2}^{\infty} \frac{1-\alpha}{[n]_{q}\left([n]_{q}-\alpha\right)} x_{n} z^{n}+\sum_{n=1}^{\infty} \frac{1-\alpha}{[n]_{q}\left([n]_{q}+\alpha\right)} \overline{y_{n} z^{n}},
$$

where $\sum_{n=2}^{\infty}\left|x_{n}\right|+\sum_{n=1}^{\infty}\left|y_{n}\right|=1$, show that the coefficient bound given by (11) is sharp.

Remark 3. When $q \rightarrow 1^{-}$, (11) reduces to the corresponding result obtained by Jahangiri [17]. Also, when $q \rightarrow 1^{-}$and $g(z) \equiv 0$, equation (11) yields to the corresponding results for analytic convex functions of order $\alpha$ obtained by Goodman [12].

In order to establish that (7) is also necessary condition, we need to define a 
class $\mathcal{T} \mathcal{H}_{q}$ consisting of functions $f=h+\bar{g}$, where

$$
h(z)=z-\sum_{n=2}^{\infty}\left|a_{n}\right| z^{n} \quad \text { and } \quad g(z)=-\sum_{n=1}^{\infty}\left|b_{n}\right| z^{n}, \quad(z \in \mathbb{D}) .
$$

The condition (11) is also necessary for the class $\mathcal{T} \mathcal{S}_{\mathcal{H}_{q}}^{\mathcal{C}}(\alpha)$ defined by $\mathcal{T} \mathcal{S}_{\mathcal{H}_{q}}^{\mathcal{C}}(\alpha):=$ $\mathcal{T H}_{q} \cap \mathcal{S}_{\mathcal{H}_{q}}^{\mathcal{C}}(\alpha)$. In fact, we prove the following coefficient characterization.

Theorem 4. Let $f=h+\bar{g}$ be defined by (13), where $f$ belongs to $\mathcal{T H}_{q}$. Then $f \in \mathcal{T S}_{\mathcal{H}_{q}}^{\mathcal{C}}(\alpha)$ if and only if

$$
\sum_{n=1}^{\infty}\left([n]_{q} \frac{[n]_{q}-\alpha}{1-\alpha}\left|a_{n}\right|+[n]_{q} \frac{[n]_{q}+\alpha}{1-\alpha}\left|b_{n}\right|\right) \leq 2,
$$

where $a_{1}=1, q \in(0,1)$ and $\alpha \in[0,1)$.

Proof. Since $\mathcal{T} \mathcal{S}_{\mathcal{H}_{q}}^{\mathcal{C}}(\alpha) \subset \mathcal{S}_{\mathcal{H}_{q}}^{\mathcal{C}}(\alpha)$, we only need to prove the "only if" part of this theorem. To this end, for functions $f=h+\bar{g}$ of the form (13), we notice that the condition (6) is equivalent to

$$
\operatorname{Re}\left\{\frac{(1-\alpha) z-\sum_{n=2}^{\infty}[n]_{q}\left([n]_{q}-\alpha\right)\left|a_{n}\right| z^{n}-\sum_{n=1}^{\infty}[n]_{q}\left([n]_{q}+\alpha\right)\left|b_{n}\right| \bar{z}^{n}}{z-\sum_{n=2}^{\infty}[n]_{q}\left|a_{n}\right| z^{n}+\sum_{n=1}^{\infty}[n]_{q}\left|b_{n}\right| \bar{z}^{n}}\right\} \geq 0 .
$$

The above required condition (15) must hold for all values of $z \in \mathbb{D},|z|=r<1$. By choosing the values of $z$ on the positive real axis where $0 \leq|z|=r<1$, we must have

$$
\frac{(1-\alpha)-\sum_{n=2}^{\infty}[n]_{q}\left([n]_{q}-\alpha\right)\left|a_{n}\right| r^{n-1}-\sum_{n=1}^{\infty}[n]_{q}\left([n]_{q}+\alpha\right)\left|b_{n}\right| r^{n-1}}{1-\sum_{n=2}^{\infty}[n]_{q}\left|a_{n}\right| r^{n-1}+\sum_{n=1}^{\infty}[n]_{q}\left|b_{n}\right| r^{n-1}} \geq 0 .
$$

If the condition (14) does not hold, then the numerator in (16) is negative for $r$ sufficiently close to 1 . Thus there exists a point $z_{0}=r_{0}$ in $(0,1)$ for which the quotient in (16) is negative. This contradicts the required condition for $f \in \mathcal{T S}_{\mathcal{H}_{q}}^{\mathcal{C}}(\alpha)$ and so the proof is complete. 


\section{Extreme Points, Convolution and Convex Combinations}

In this section, we first determine the extreme points of the closed convex hulls of $\mathcal{T S}_{\mathcal{H}_{q}}^{\mathcal{C}}(\alpha)$, denoted by $\operatorname{clco} \mathcal{T} \mathcal{S}_{\mathcal{H}_{q}}^{\mathcal{C}}(\alpha)$.

Theorem 5. Let $f$ be given by (13). Then $f \in \operatorname{clco} \mathcal{T S}_{\mathcal{H}_{q}}^{\mathcal{C}}(\alpha)$ if and only if $f(z)=$ $\sum_{n=1}^{\infty}\left(x_{n} h_{n}(z)+y_{n} g_{n}(z)\right)$, where

$$
\begin{gathered}
h_{1}(z)=z, \quad h_{n}(z)=z-\frac{1-\alpha}{[n]_{q}\left([n]_{q}-\alpha\right)} z^{n}, \quad(n \geq 2) \\
g_{n}(z)=z-\frac{1-\alpha}{[n]_{q}\left([n]_{q}+\alpha\right)} \bar{z}^{n}, \quad(n \geq 1)
\end{gathered}
$$

and $\sum_{n=1}^{\infty}\left(x_{n}+y_{n}\right)=1$ where $x_{n} \geq 0$ and $y_{n} \geq 0$. In particular, the extreme points of $\mathcal{T} \mathcal{S}_{\mathcal{H}_{q}}^{\mathcal{C}}(\alpha)$ are $\left\{h_{n}\right\}$ and $\left\{g_{n}\right\}$.

Proof. For a function $f$ of the form $f(z)=\sum_{n=1}^{\infty}\left(x_{n} h_{n}(z)+y_{n} g_{n}(z)\right)$, where $\sum_{n=1}^{\infty}\left(x_{n}+\right.$ $\left.y_{n}\right)=1$, we obtain

$$
f(z)=z-\sum_{n=2}^{\infty} \frac{1-\alpha}{[n]_{q}\left([n]_{q}-\alpha\right)} x_{n} z^{n}-\sum_{n=1}^{\infty} \frac{1-\alpha}{[n]_{q}\left([n]_{q}+\alpha\right)} y_{n} \bar{z}^{n} .
$$

Then $f \in \operatorname{clco} \mathcal{T} \mathcal{S}_{\mathcal{H}_{q}}^{\mathcal{C}}(\alpha)$ because

$$
\begin{gathered}
\sum_{n=2}^{\infty} \frac{[n]_{q}\left([n]_{q}-\alpha\right)}{1-\alpha}\left(\frac{1-\alpha}{[n]_{q}\left([n]_{q}-\alpha\right)} x_{n}\right)+\sum_{n=1}^{\infty} \frac{[n]_{q}\left([n]_{q}+\alpha\right)}{1-\alpha}\left(\frac{1-\alpha}{[n]_{q}\left([n]_{q}+\alpha\right)} y_{n}\right) \\
=\sum_{n=2}^{\infty} x_{n}+\sum_{n=1}^{\infty} y_{n}=1-x_{1} \leq 1 .
\end{gathered}
$$

Conversely, suppose $f \in \operatorname{clco} \mathcal{T} \mathcal{S}_{\mathcal{H}_{q}}^{\mathcal{C}}(\alpha)$. In view of $(14)$, we have $\left|a_{n}\right| \leq \frac{1-\alpha}{[n]_{q}\left([n]_{q}-\alpha\right)}$ and $\left|b_{n}\right| \leq \frac{1-\alpha}{[n]_{q}\left([n]_{q}+\alpha\right)}$. Set

$$
x_{n}=\frac{[n]_{q}\left([n]_{q}-\alpha\right)}{1-\alpha}\left|a_{n}\right|,(n \geq 2) \quad \text { and } \quad y_{n}=\frac{[n]_{q}\left([n]_{q}+\alpha\right)}{1-\alpha}\left|b_{n}\right|,(n \geq 1) .
$$

By Theorem 4, $\sum_{n=2}^{\infty} x_{n}+\sum_{n=1}^{\infty} y_{n} \leq 1$. Therefore we define $x_{1}=1-\sum_{n=2}^{\infty} x_{n}-\sum_{n=1}^{\infty} y_{n} \geq 0$. Consequently, we obtain $f(z)=\sum_{n=1}^{\infty}\left(x_{n} h_{n}(z)+y_{n} g_{n}(z)\right)$ as required. 
Using definition of convolution, we show that the class $\mathcal{T S}_{\mathcal{H}_{q}}^{\mathcal{C}}(\alpha)$ is closed under convolution.

Theorem 6. For $0 \leq \beta<\alpha<1$, suppose $f \in \mathcal{T S}_{\mathcal{H}_{q}}^{\mathcal{C}}(\alpha)$ and $F \in \mathcal{T S}_{\mathcal{H}_{q}}^{\mathcal{C}}(\beta)$. Then $f * F \in \mathcal{T S}_{\mathcal{H}_{q}}^{\mathcal{C}}(\alpha) \subset \mathcal{T} \mathcal{S}_{\mathcal{H}_{q}}^{\mathcal{C}}(\beta)$.

Proof. Let $f(z)=z-\sum_{n=2}^{\infty}\left|a_{n}\right| z^{n}-\sum_{n=1}^{\infty}\left|b_{n}\right| \bar{z}^{n}$ and $F(z)=z-\sum_{n=2}^{\infty}\left|A_{n}\right| z^{n}-\sum_{n=1}^{\infty}\left|B_{n}\right| \bar{z}^{n}$, then

$$
(f * F)(z)=z+\sum_{n=2}^{\infty}\left|a_{n}\right|\left|A_{n}\right| z^{n}+\sum_{n=1}^{\infty}\left|b_{n}\right|\left|B_{n}\right| \bar{z}^{n} .
$$

Since of $F \in \mathcal{T S}_{\mathcal{H}_{q}}^{\mathcal{C}}(\beta)$, it follows from Theorem 4 that $\left|A_{n}\right| \leq 1$ and $\left|B_{n}\right| \leq 1$. Therefore, we have

$$
\begin{aligned}
& \sum_{n=2}^{\infty} \frac{[n]_{q}\left([n]_{q}-\alpha\right)}{1-\alpha}\left|a_{n}\right|\left|A_{n}\right|+\sum_{n=1}^{\infty} \frac{[n]_{q}\left([n]_{q}+\alpha\right)}{1-\alpha}\left|b_{n}\right|\left|B_{n}\right| \\
& \leq \sum_{n=2}^{\infty} \frac{[n]_{q}\left([n]_{q}-\alpha\right)}{1-\alpha}\left|a_{n}\right|+\sum_{n=1}^{\infty} \frac{[n]_{q}\left([n]_{q}+\alpha\right)}{1-\alpha}\left|b_{n}\right| \leq 1 .
\end{aligned}
$$

In view of Theorem 4 , it follows that $f * F \in \mathcal{T S}_{\mathcal{H}_{q}}^{\mathcal{C}}(\alpha) \subset \mathcal{T} \mathcal{S}_{\mathcal{H}_{q}}^{\mathcal{C}}(\beta)$.

We now prove that $\mathcal{T} \mathcal{S}_{\mathcal{H}_{q}}^{\mathcal{C}}(\alpha)$ is closed under convex combination of its members.

Theorem 7. The class $\mathcal{T S}_{\mathcal{H}_{q}}^{\mathcal{C}}(\alpha)$ is closed under convex combination.

Proof. For $j=1,2,3, .$. , let $f_{j} \in \mathcal{T} \mathcal{S}_{\mathcal{H}_{q}}^{\mathcal{C}}(\alpha)$, where $f_{j}$ is given by

$$
f_{j}(z)=z-\sum_{n=2}^{\infty}\left|a_{n_{j}}\right| z^{n}-\sum_{n=1}^{\infty}\left|b_{n_{j}}\right| \bar{z}^{n} .
$$

Then, by Theorem 4

$$
\sum_{n=1}^{\infty}\left(\frac{[n]_{q}\left([n]_{q}-\alpha\right)}{1-\alpha}\left|a_{n_{j}}\right|+\frac{[n]_{q}\left([n]_{q}+\alpha\right)}{1-\alpha}\left|b_{n_{j}}\right|\right) \leq 2 .
$$

For $\sum_{j=1}^{\infty} t_{j}=1,0 \leq t_{j} \leq 1$, the convex combination of $f_{j}$ may be written as

$$
\sum_{j=1}^{\infty} t_{j} f_{j}(z)=z-\sum_{n=2}^{\infty} \sum_{j=1}^{\infty} t_{j}\left|a_{n_{j}}\right| z^{n}-\sum_{n=1}^{\infty} \sum_{j=1}^{\infty} t_{j}\left|b_{n_{j}}\right| \bar{z}^{n}
$$


Using (17), we have

$$
\begin{aligned}
& \sum_{n=1}^{\infty}\left(\frac{[n]_{q}\left([n]_{q}-\alpha\right)}{1-\alpha} \sum_{j=1}^{\infty} t_{j}\left|a_{n_{j}}\right|+\frac{[n]_{q}\left([n]_{q}+\alpha\right)}{1-\alpha} \sum_{j=1}^{\infty} t_{j}\left|b_{n_{j}}\right|\right) \\
& =\sum_{j=1}^{\infty} t_{j} \sum_{n=1}^{\infty}\left(\frac{[n]_{q}\left([n]_{q}-\alpha\right)}{1-\alpha}\left|a_{n_{j}}\right|+\frac{[n]_{q}\left([n]_{q}+\alpha\right)}{1-\alpha}\left|b_{n_{j}}\right|\right) \\
& \leq 2 \sum_{j=1}^{\infty} t_{j}=2
\end{aligned}
$$

and so by Theorem 4 , we have $\sum_{j=1}^{\infty} t_{j} f_{j}(z) \in \mathcal{T S}_{\mathcal{H}_{q}}^{\mathcal{C}}(\alpha)$.

\section{Distortion Bounds and Covering Theorem}

The following theorem gives distortion bounds for functions in $\mathcal{T} \mathcal{S}_{\mathcal{H}_{q}}^{\mathcal{C}}(\alpha)$, which yields covering result for this class.

Theorem 8. If $f \in \mathcal{T S}_{\mathcal{H}_{q}}^{\mathcal{C}}(\alpha)$, then for $|z|=r<1$ we have

$$
|f(z)| \leq\left(1+\left|b_{1}\right|\right) r+\frac{1}{[2]_{q}}\left(\frac{1-\alpha}{[2]_{q}-\alpha}-\frac{1+\alpha}{[2]_{q}-\alpha}\left|b_{1}\right|\right) r^{2}
$$

and

$$
|f(z)| \geq\left(1-\left|b_{1}\right|\right) r-\frac{1}{[2]_{q}}\left(\frac{1-\alpha}{[2]_{q}-\alpha}-\frac{1+\alpha}{[2]_{q}-\alpha}\left|b_{1}\right|\right) r^{2} .
$$

Proof. Let $\mathcal{T S}_{\mathcal{H}_{q}}^{\mathcal{C}}(\alpha)$. Taking the absolute value of $f$, we obtain

$$
\begin{aligned}
|f(z)| & \leq\left(1+\left|b_{1}\right|\right) r+\sum_{n=2}^{\infty}\left(\left|a_{n}\right|+\left|b_{n}\right|\right) r^{n} \\
& \leq\left(1+\left|b_{1}\right|\right) r+\sum_{n=2}^{\infty}\left(\left|a_{n}\right|+\left|b_{n}\right|\right) r^{2} \\
& \leq\left(1+\left|b_{1}\right|\right) r+\frac{1-\alpha}{[2]_{q}\left([2]_{q}-\alpha\right)} \sum_{n=2}^{\infty}\left(\frac{[2]_{q}\left([2]_{q}-\alpha\right)}{1-\alpha}\left|a_{n}\right|+\frac{[2]_{q}\left([2]_{q}-\alpha\right)}{1-\alpha}\left|b_{n}\right|\right) r^{2} \\
& \leq\left(1+\left|b_{1}\right|\right) r+\frac{1-\alpha}{[2]_{q}\left([2]_{q}-\alpha\right)} \sum_{n=2}^{\infty}\left(\frac{[n]_{q}\left([n]_{q}-\alpha\right)}{1-\alpha}\left|a_{n}\right|+\frac{[n]_{q}\left([n]_{q}+\alpha\right)}{1-\alpha}\left|b_{n}\right|\right) r^{2} \\
& \leq\left(1+\left|b_{1}\right|\right) r+\frac{1-\alpha}{[2]_{q}\left([2]_{q}-\alpha\right)}\left(1-\frac{1+\alpha}{1-\alpha}\left|b_{1}\right|\right) r^{2} .
\end{aligned}
$$


This proves (18). We omit the proof of (19) because its proof similar to (18).

The following covering result follows from the inequality (19).

Corollary 9. If $f \in \mathcal{T} \mathcal{S}_{\mathcal{H}_{q}}^{\mathcal{C}}(\alpha)$, then

$$
\left\{w:|w|<\frac{[2]_{q}\left([2]_{q}-\alpha\right)-1+\alpha+\left(1+\alpha-[2]_{q}\left([2]_{q}-\alpha\right)\right)\left|b_{1}\right|}{[2]_{q}\left([2]_{q}-\alpha\right)}\right\} \subset f(\mathbb{D}) .
$$

Remark 4. For $q \rightarrow 1^{-}$, the covering theorem in Corollary 9 yields the corresponding traditional result for harmonic convex functions of order $\alpha$ obtained in [17].

Moreover, for $q \rightarrow 1^{-}, \alpha=b_{1}=0$, Corollary 9 yields the following result given in [17].

Remark 5. If $f \in \mathcal{T}^{0} \mathcal{S}_{\mathcal{H}}^{\mathcal{C}}(0)$, then $\{w:|w|<3 / 4\} \subset f(\mathbb{D})$.

\section{6. $q-$ Bernardi Harmonic Integral Operator}

In this section, we prove that the class $\mathcal{T S}_{\mathcal{H}_{q}}^{\mathcal{C}}(\alpha)$ is closed under $q$-Bernardi harmonic integral operator given by (5).

Theorem 10. Let $f=h+\bar{g} \in \mathcal{T} \mathcal{H}_{q}$ be given by (13) and $f \in \mathcal{T S}_{\mathcal{H}_{q}}^{\mathcal{C}}(\alpha)$, then $F$ defined by (5) also belongs to $\mathcal{T S}_{\mathcal{H}_{q}}^{\mathcal{C}}(\alpha)$.

Proof. From (5), it follows that

$$
F(z)=z-\sum_{n=2}^{\infty}\left(\frac{[c+1]_{q}}{[c+n]_{q}}\right)\left|a_{n}\right| z^{n}-\sum_{n=1}^{\infty}\left(\frac{[c+1]_{q}}{[c+n]_{q}}\right)\left|b_{n}\right| \bar{z}^{n} .
$$

Since $f \in \mathcal{T S}_{\mathcal{H}_{q}}^{\mathcal{C}}(\alpha)$, it follows that the inequality (14) is equivalent to

$$
\sum_{n=2}^{\infty}[n]_{q} \frac{[n]_{q}-\alpha}{1-\alpha}\left|a_{n}\right|+\sum_{n=1}^{\infty}[n]_{q} \frac{[n]_{q}+\alpha}{1-\alpha}\left|b_{n}\right| \leq 1 .
$$

Therefore

$$
\begin{aligned}
& \sum_{n=2}^{\infty}[n]_{q} \frac{[n]_{q}-\alpha}{1-\alpha}\left(\frac{[c+1]_{q}}{[c+n]_{q}}\left|a_{n}\right|\right)+\sum_{n=1}^{\infty}[n]_{q} \frac{[n]_{q}+\alpha}{1-\alpha}\left(\frac{[c+1]_{q}}{[c+n]_{q}}\left|b_{n}\right|\right) \\
& \leq \sum_{n=2}^{\infty}[n]_{q} \frac{[n]_{q}-\alpha}{1-\alpha}\left|a_{n}\right|+\sum_{n=1}^{\infty}[n]_{q} \frac{[n]_{q}+\alpha}{1-\alpha}\left|b_{n}\right| \\
& \leq 1 .
\end{aligned}
$$

Thus $F \in \mathcal{T} \mathcal{S}_{\mathcal{H}_{q}}^{\mathcal{C}}(\alpha)$, by Theorem 4 . 
O. P. Ahuja, A. Çetinkaya, Y. Polatoğlu - Harmonic Univalent Convex ...

\section{REFERENCES}

[1] Om P. Ahuja, Planar harmonic univalent and related mappings, J. Inequal. Pure Appl. Math. 6(4) (2005), Article 122, 18pp.

[2] Om P. Ahuja, Recent advances in the theory of harmonic univalent mappings in the plane, Math. Student 83(1-4) (2014), 125-154.

[3] Om P. Ahuja, J. M. Jahangiri, H. Silverman, Convolutions for special classes of harmonic univalent functions, Appl. Math. Lett. 16(6) (2003), 905-909.

[4] Om P. Ahuja, A. Çetinkaya, Y. Polatog̃lu, Bieberbach-de Branges and FeketeSzegö inequalities for certain families of $q$-convex and $q$-close-to-convex functions, J. Comput. Anal. Appl. 26(4) (2019), 639-649.

[5] G. E. Andrews, Applications of basic hypergeometric functions, SIAM Rev. 16(4) (1974), 441-484.

[6] A. Aral, V. Gupta, R. P. Agarwal, Applications of $q$-calculus in operator theory, Springer, New-York, 2013. ISBN:978-1-4614-6945-2; 978-1-4614-6946-9.

[7] J. Clunie, T. Sheil-Small, Harmonic univalent functions, Ann. Acad. Sci. Fenn. Ser. A. I. Math. 9 (1984), 3-25.

[8] P. Duren, Harmonic mappings in the plane, Cambridge Tracts in Math.V156, Cambridge University Press, 2004. ISBN: 0-521-64121-7.

[9] T. Ernst, A method for q-calculus, Journal of Nonlinear Mathematical Physics, 10(4) (2003), 487-525.

[10] N. J. Fine, Basic hypergeometric series and applications, Math. Surveys Monogr., No. 7, Amer. Math. Soc., Providence, 1988.

[11] G. Gasper, M. Rahman, Basic hypergeometric series, Cambridge University Press, 2004.

[12] A. W. Goodman, Univalent functions and non-analytic curves, Proc. Amer. Math. Soc. 8(3) (1957), 598-601.

[13] M. E. H. Ismail, E. Merkes, D. Steyr, A generalization of starlike functions, Complex Variables Theory Appl. 14(1) (1990), 77-84.

[14] F. H. Jackson, On q-functions and a certain difference operator, Trans. Royal Soc. Edinburgh, 46(2) (1909), 253-281.

[15] F. H. Jackson, On q-definite integrals, Quart. J. Pure Appl. Math. 41 (1910), 193-203.

[16] F. H. Jackson, q-difference equations, Amer. J. Math. 32(4) (1910), 305-314.

[17] J. M. Jahangiri, Coefficient bounds and univalence criteria for harmonic functions with negative coefficients, Ann. Univ. Marie Curie-Sklodowska Sect. A, 52(2) (1998), 57-66. 
[18] V. Kac, P. Cheung, Quantum calculus, Springer-Verlag, New-York, 2002.

[19] R. J. Libera, Some classes of regular univalent functions, Proc. Amer. Math. Soc. 16(4) (1965), 755-758.

[20] K. I. Noor, S. Riaz, M. A. Noor, On q-Bernardi integral operator, TWMS J. Pure Math. 8(1) (2017), 3-11.

[21] S. Porwal, M. K. Aouf, On a new subclass of harmonic univalent functions defined by fractional calculus operator, Journal of Fractional Calculus ans Applications, 4(10) (2013), 1-12.

[22] M. S. Robertson, On the theory of univalent functions, Ann. of Math. 37(2) (1936), 374-408.

[23] T. M. Seoudy, M. K. Aouf, Coefficient estimates of new classes of $q$-starlike and q-convex functions of complex order, J. Math. Inequal. 10(1) (2016), 135-145.

Om P. Ahuja

Department of Mathematical Sciences, Kent State University

Ohio, 44021, U.S.A

email: oahuja@kent.edu

Asena Çetinkaya

Department of Mathematics and Computer Sciences, Istanbul Kültür University Istanbul, Turkey

email: asnfigen@hotmail.com

Yaşar Polatog̃lu

Department of Mathematics and Computer Sciences, Istanbul Kültür University Istanbul, Turkey

email: y.polatoglu@iku.edu.tr 